\title{
A Relative Total Cost Index for the Evaluation of Transportation Network Robustness in the Presence of Degradable Links and Alternative Travel Behavior
}

\author{
Anna Nagurney and Qiang Qiang \\ Department of Finance and Operations Management \\ Isenberg School of Management \\ University of Massachusetts \\ Amherst, Massachusetts 01003 \\ October 2007; revised April 2008 \\ International Transactions in Operational Research (2009) 16, pp. 49-67.
}

\begin{abstract}
In this paper, we demonstrate how to capture the robustness of a transportation network in the case of degradable links represented by decreasing capacities. In particular, with the use of Bureau of Public Road user link travel cost functions, we propose two relative total cost indices to assess transportation network robustness in the case of travel behavior associated with either user-optimization or system-optimization. We derive upper bounds of the relative total cost index for transportation networks with special structure and congestion parameters under the user-optimal flow pattern. We also derive an upper bound for the relative total cost index under the system-optimal flow pattern for general transportation networks. Numerical examples are presented for illustration purposes.

This research is the first to quantify transportation network robustness in the presence of degradable links and alternative travel behavior. This research has implication for transportation planning and management, for vulnerability analysis, as well as for security issues.
\end{abstract}

Key words: transportation networks, urban transportation, freight networks, network robustness, relative total cost index, system-optimization, user-optimization, Bureau of Public Roads link cost functions, degradable links, vulnerability analysis 


\section{Introduction}

In this paper, we propose a novel index for evaluating the robustness of a transportation network based on the relative total cost of the transportation network in the case of link degradations captured through a uniform link capacity ratio. The relative total cost index can be evaluated at either user-optimal (U-O) traffic flows or system-optimal (S-O) traffic flows. In particular, we consider congested urban transportation networks, which can also include freight traffic in terms of trucks, in which the user link cost functions are of the general Bureau of Public Roads (1964) (BPR) form, which is widely used in practice. Such a functional form contains the practical capacity of a link implicitly. The new index enables the quantitative assessment of the changes in the relative total cost of a transportation network, in the case of alternative travel behavior, when the link practical capacities are decreased.

The degradation of transportation networks due to poor maintenance, natural disasters, deterioration over time, as well as unforeseen attacks now lead to estimates of $\$ 94$ billion in the United States in terms of needed repairs for roads alone (cf. American Society of Civil Engineers, 2005). Poor road conditions in the United States cost US motorists $\$ 54$ billion in repairs and operating costs annually. Moreover, due to the constant breakdowns of the US transportation networks, American commuters now spend 3.5 billion hours a year stuck in traffic, which translates to a cost of $\$ 63.2$ billion a year to the economy (cf. American Society of Civil Engineers, 2005). Even worse, over one-quarter of the nation's 590,750 bridges were rated structurally deficient or functionally obsolete (National Bridge Inventory, 2005). At the same time, a recent report from the Federal Highway Administration (2006) states that the United States is experiencing a freight capacity crisis that threatens the strength and productivity of the US economy. According to the American Road \& Transportation Builders Association (see Jeanneret, 2006), nearly $75 \%$ of US freight is carried in the US on highways and bottlenecks are causing truckers 243 million hours of delay annually with an estimated associated cost of $\$ 8$ billion. It is noted that the US government is facing a $\$ 1.6$ trillion deficit over the five years in terms of infrastructure repairing and reconstruction according to a recent estimate (Environment News Service, 2008).

Hence, the construction of suitable transportation network indices that can assist in the evaluation of transportation network robustness is timely and of both theoretical and practical importance. In the era of an ailing U.S. infrastructure, we believe that to study transportation network robustness by analyzing capacity degradation is especially relevant in order to prevent such disasters as the Minneapolis Bridge Collapse (cf. Wald and Chang, 2007). 
Although the rigorous modeling and analysis of transportation networks and the underlying behavior of travelers dates to the seminal book of Beckmann, McGuire, and Winsten (1956), the research into the robustness of transportation networks in the presence of disruptions is relatively recent. To the best of our knowledge, the papers of Sakakibara et al. (2004) and of Scott et al. (2006) stand as the first attempts to address the robustness of transportation networks. Sakakibara et al. (2004) proposed a topological index and considered a transportation network to be robust if it is "dispersed" in terms of the number of links connected to each node. Scott et al. (2006), on the other hand, examined transportation network robustness by computing the increase in the total network cost evaluated at the U-O flow pattern after the removal of each link. In particular, Scott et al. (2006) sought to identify the critical network links. We believe that, as suggested by the definition of robustness (see the discussion that follows in this section), it is also relevant to study transportation network robustness from the point of view of the network performance drop due to the partial degradation of links caused by disruptions or network infrastructure deteriorations. Furthermore, since system-optimization and user-optimization are the central concepts in the modeling and analysis of transportation networks, it is imperative to study network robustness from both perspectives. Therefore, the approach proposed in this paper is unique since it allows for alternative travel behaviors as well as degradations in the network links, rather than the explicit removal of the links (or nodes).

We note that Nagurney and Qiang (2007a) (see also the references therein) proposed earlier a network efficiency measure that allows for the importance identification and ranking of network components and their approach is not limited to the removal of a single link but, rather, the network efficiency measure can handle the impact of the removal of subsets of network components, whether nodes or links, or combinations thereof. Furthermore, based on the network efficiency measure of Nagurney and Qiang (2007a), the authors proposed a transportation network robustness measure to study the network efficiency loss when facing link capacity degradations under the user-optimization behavior (Nagurney and Qiang, 2007b). In this paper, however, we extend the above research to study different user behaviors and from the point of view of total network cost.

In addition, in this paper, our goal is to study transportation robustness with capacity degradation under different user behaviors, which is different from the goal of research concerning the "price of anarchy" (cf. Roughgarden, 2005, and the references therein). The price of anarchy is defined as the ratio of the total cost evaluated at the U-O solution and the total cost evaluated at the S-O solution and the emphasis has been principally in the context of Internet-based applications. Upper bounds on the price of anarchy have been 
derived for various user link cost functional forms; see Roughgarden (2005) for separable user link cost functions and Perakis (2007) for asymmetric ones. As discussed above, we are concerned with transportation network robustness, and, hence, we focus on the widely used in practice BPR link cost functional form.

System robustness has been studied in both computer science and in engineering. According to the Institute of Electrical and Electronic Engineers (1990), robustness can be defined as "the degree to which a system or component can function correctly in the presence of invalid inputs or stressful environmental conditions." Gribble (2001) defined system robustness as "the ability of a system to continue to operate correctly across a wide range of operational conditions, and to fail gracefully outside of that range." Schillo et al. (2001) argued that robustness has to be studied "in relation to some definition of performance measure." According to Holmgren (2007), "Robustness signifies that the system will retain its system structure (function) intact (remain unchanged or nearly unchanged) when exposed to perturbations." The physics literature on complex networks has also investigated issues of robustness but it has focused principally on the specific network topologies and the explicit elimination of nodes in the network (see Albert, Jeong, and Barabási, 2000).

This paper is organized as follows. In Section 2, we briefly recall the well-known U-O and S-O transportation network models corresponding, respectively, to Wardrop's first and second principles of travel behavior (cf. Wardrop, 1952; see also, e.g., Beckmann, McGuire and Winsten, 1956, Dafermos and Sparrow, 1969, Smith, 1979, Dafermos, 1980, Sheffi, 1985, and Nagurney, 2000). Recall (cf. Dafermos and Sparrow, 1969) that a flow pattern is said to be U-O if all used paths, that is, those with positive flow, connecting each origin/destination pair of nodes in a network have user travel costs that are equal and minimal. A flow pattern is said to be S-O if it minimizes the total cost in a network, in which case, the Kuhn-Tucker optimality conditions (cf. Bazaraa, Sherali, and Shetty, 1993) coincide with the statement that all used paths connecting each origin/destination pair of nodes have marginal total costs that are equal and minimal. In Section 2, we propose the relative total cost index that can be used to assess transportation network robustness and which permits either U-O or S-O travel behavior. For completeness, we also present the relationship between the ratio of the proposed indices and the price of anarchy.

In Section 3, we derive some theoretical results. In particular, we first prove that for certain networks of special structure, with links characterized by user link cost functions of BPR form, and with identical free flow travel terms, the relative total cost index is identical under the U-O and the S-O flow patterns. We then show that, for the same network topologies and with user link cost functions also of the BPR form, but linear, that 
the relative total cost index under the U-O flow pattern can be obtained via an explicit formula. In addition, we derive an upper bound for the relative total cost index under the U-O flow pattern. Finally, we derive an upper bound for the relative total cost index for transportation network robustness, under the S-O flow pattern, for any transportation network in the case of user link cost functions of BPR form.

In Section 4, we provide several numerical examples, including the Sioux-Falls network example, and we relate them to the derived theoretical results, where applicable. In Section 5 , we present a summary of the results along with our conclusions. 


\section{The Relative Total Cost Index for Assessing Transportation Network Robust- ness Under Distinct Travel Behaviors}

In this Section, we present the relative total cost index for assessing transportation network robustness under distinct travel behaviors and in the presence of degradable network links. The user link cost functions are assumed to be of the BPR functional form in which the practical link capacity is incorporated.

We consider a network $G$ with the set of directed links $L$ with $n_{L}$ elements and the set of origin/destination (O/D) pairs $W$ with $n_{W}$ elements. We denote the set of acyclic paths joining $\mathrm{O} / \mathrm{D}$ pair $w$ by $P_{w}$. The set of (acyclic) paths for all $\mathrm{O} / \mathrm{D}$ pairs is denoted by $P$ and there are $n_{P}$ paths in the network. Links are denoted by $a, b$, etc; paths by $p, q$, etc., and $\mathrm{O} / \mathrm{D}$ pairs by $w_{1}, w_{2}$, etc.

We assume that the demand $d_{w}$ is known for all $\mathrm{O} / \mathrm{D}$ pairs $w \in W$. We denote the nonnegative flow on path $p$ by $x_{p}$ and the flow on link $a$ by $f_{a}$ and we group the path flows into the vector $x \in R_{+}^{n_{P}}$ and the link flows into the vector $f \in R_{+}^{n_{L}}$.

The following conservation of flow equations must hold:

$$
\sum_{p \in P_{w}} x_{p}=d_{w}, \quad \forall w \in W
$$

which means that the sum of path flows on paths connecting each O/D pair must be equal to the demand for that $\mathrm{O} / \mathrm{D}$ pair.

The link flows are related to the path flows, in turn, through the following conservation of flow equations:

$$
f_{a}=\sum_{p \in P} x_{p} \delta_{a p}, \quad \forall a \in L,
$$

where $\delta_{a p}=1$, if link $a$ is contained in path $p$, and $\delta_{a p}=0$, otherwise. Hence, the flow on a link is equal to the sum of the flows on paths that contain that link.

The user (travel) cost on a link $a$ is denoted by $c_{a}$ where

$$
c_{a}=c_{a}\left(f_{a}\right), \quad \forall a \in L
$$

We assume that the link cost functions are continuous and monotonically increasing.

The user (travel) cost on a path $p$ is denoted by $C_{p}$. The user costs on paths are related to user costs on links through the following equations:

$$
C_{p}=\sum_{a \in L} c_{a} \delta_{a p}, \quad \forall p \in P,
$$


that is, the user cost on a path is equal to the sum of user costs on links that make up the path. In engineering practice (see Sheffi, 1985), the travel time on a link is used as a proxy for the travel cost.

In view of (1) - (4), we may write

$$
C_{p}=C_{p}(x), \quad \forall p \in P
$$

In this paper, we will consider user link cost functions known as BPR functions, given by

$$
c_{a}\left(f_{a}\right)=t_{a}^{0}\left[1+k\left(\frac{f_{a}}{u_{a}}\right)^{\beta}\right], \quad \forall a \in L,
$$

where $f_{a}$ is the flow on link $a ; u_{a}$ is the "practical" capacity on link $a$, which also has the interpretation of the level-of-service flow rate; $t_{a}^{0}$ is the free-flow travel time or cost on link $a ; k$ and $\beta$ are the model parameters and both take on positive values (Bureau of Public Roads, 1964, and Sheffi, 1985). Often in applications $k=.15$ and $\beta=4$.

The total cost on a link $a$, denoted by $\hat{c}_{a}$, is given by:

$$
\hat{c}_{a}=\hat{c}_{a}\left(f_{a}\right)=c_{a}\left(f_{a}\right) \times f_{a}=t_{a}^{0}\left[1+k\left(\frac{f_{a}}{u_{a}}\right)^{\beta}\right] \times f_{a}, \quad \forall a \in L .
$$

The total cost on a network, denoted by $T C$, is, hence, given by:

$$
T C=\sum_{a \in L} \hat{c}_{a}\left(f_{a}\right)
$$

where the link flows $f$ must lie in the feasible set $\mathcal{K}: \mathcal{K} \equiv\left\{f \in R_{+}^{n_{L}} \mid x \in R_{+}^{n_{P}}\right.$ satisfying (1), (2) $\}$.

\subsection{The User-Optimal Traffic Flow Pattern}

A U-O solution is defined as follows. A path flow pattern $x^{*} \in \mathcal{K}^{1}$, where $\mathcal{K}^{1} \equiv\{x \mid x \in$ $R_{+}^{n_{P}}$ and (1) holds $\}$, is said to be a U-O traffic flow pattern, if the following conditions hold for each O/D pair $w \in W$ and each path $p \in P_{w}$ :

$$
C_{p}\left(x^{*}\right) \begin{cases}=\lambda_{w}, & \text { if } \quad x_{p}^{*}>0 \\ \geq \lambda_{w}, & \text { if } \quad x_{p}^{*}=0 .\end{cases}
$$

The interpretation of conditions (9) is that all used paths connecting an O/D pair $w$ have equal and minimal user travel costs (with the minimal path costs equal to the equilibrium travel disutility, denoted by $\lambda_{w}$ ). These conditions are also referred to as the user-optimized conditions (cf. Dafermos and Sparrow, 1969). In this classical transportation network equilibrium problem, in which the cost on each link (cf. (3)) depends solely on the flow on that 
link, the U-O (equivalently, transportation network equilibrium) conditions (9) can be reformulated as the solution to an appropriately constructed optimization problem, as established in Beckmann, McGuire, and Winsten (1956). Indeed, the equilibrium link flow (and path flow pattern) can be obtained via the solution of the following optimization problem:

$$
\operatorname{Minimize}_{f \in \mathcal{K}} \sum_{a \in L} \int_{0}^{f_{a}} c_{a}(y) d y .
$$

For additional background on this model, along with its impacts, see Boyce, Mahmassani, and Nagurney (2005). In particular, we know that if the user link cost functions are strictly monotone (cf. Nagurney, 1999) then the U-O link flow pattern is unique.

\subsection{The System-Optimal Traffic Flow Pattern}

We now turn to the system-optimization problem in which a central controller seeks to minimize the total cost in a transportation network system expressed as (see (8)):

$$
\operatorname{Minimize}_{f \in \mathcal{K}} \sum_{a \in L} \hat{c_{a}}\left(f_{a}\right) \text {. }
$$

Under the assumption of increasing user link cost functions, the optimality conditions, which correspond to the Kuhn-Tucker conditions (see Bazaraa, Sherali, and Shetty, 1993), are: for each path $p \in P_{w}$, and every O/D pair $w, x$ is a S-O path flow pattern if $x$ satisfies:

$$
\hat{C}_{p}^{\prime}(x) \begin{cases}=\mu_{w}, & \text { if } \quad x_{p}>0 \\ \geq \mu_{w}, & \text { if } \quad x_{p}=0\end{cases}
$$

where $\hat{C}_{p}^{\prime}(x)$ denotes the marginal of the total cost on path $p$, given by:

$$
\hat{C}_{p}^{\prime}(x)=\sum_{a \in L} \frac{\partial \hat{c_{a}}\left(f_{a}\right)}{\partial f_{a}} \delta_{a p}, \quad \forall p \in P .
$$

Note that in the case of system-optimization, all used paths connecting an O/D pair have equal and minimal marginal total costs.

\subsection{Relative Total Cost Index for Assessing the Robustness of a Transportation Network}

We now propose an index based on the relative total cost that assesses the robustness of a transportation network based on the two behavioral solution concepts, namely, the total cost evaluated under the user-optimizing flow pattern, denoted by $T C_{U-O}$, and the systemoptimizing flow pattern, denoted-by $T C_{S-O}$, respectively. In particular, $T C_{U-O}$ denotes the 
total cost on the network as given by expression (8), where the vector $f$ is the solution to the user-optimizing (or transportation network equilibrium) conditions (9). On the other hand, $T C_{S-O}$ is the total cost on the network as given also by expression (8), but now evaluated at the flow pattern given by the solution to the S-O problem (11). We believe that the total cost is an appropriate measure since it represents the total cost to society associated with travel on transportation networks. Moreover, as the links degrade and the practical capacity of links decreases the total cost is expected to increase and, hence, the relative total cost of a transportation network reflects the robustness of the transportation network.

The relative total cost index for a transportation network $G$ with the vector of demands $d$, the vector of user link cost functions $c$, and the vector of link capacities $u$ is defined as the relative total cost increase under a given uniform capacity retention ratio $\gamma(\gamma \in(0,1])$ so that the new capacities (cf. (7)) are given by $\gamma u$. Let $c$ denote the vector of BPR user link cost functions and let $d$ denote the vector of $\mathrm{O} / \mathrm{D}$ pair travel demands. The mathematical definition of the index under the user-optimizing flow pattern, denoted by $\mathcal{I}_{U-O}^{\gamma}$, is then:

$$
\mathcal{I}_{U-O}^{\gamma}=\mathcal{I}_{U-O}(G, c, d, \gamma, u)=\frac{T C_{U-O}^{\gamma}-T C_{U-O}}{T C_{U-O}} \times 100 \%
$$

where $T C_{U-O}$ and $T C_{U-O}^{\gamma}$ are the total network costs evaluated under the user-optimizing flow pattern with the original capacities and the remaining capacities (i.e., $\gamma u$ ), respectively.

The mathematical definition of the index under the system-optimizing flow pattern is:

$$
\mathcal{I}_{S-O}^{\gamma}=\mathcal{I}_{S-O}(G, c, d, \gamma, u)=\frac{T C_{S-O}^{\gamma}-T C_{S-O}}{T C_{S-O}} \times 100 \%,
$$

where $T C_{S-O}$ and $T C_{S-O}^{\gamma}$ are the total network costs evaluated at the system-optimizing flow pattern with the original capacities and the remaining capacities (i.e., $\gamma u$ ), respectively.

For example, if $\gamma=.9$ this means that the total user link cost functions given by (7) now have the link capacities given by $.9 u_{a}$ for $a \in L$; if $\gamma=.7$ then the link capacities become $.7 u_{a}$ for all links $a \in L$, and so on.

From the above definition(s), a transportation network, under a given capacity retention/deterioration ratio $\gamma$ (and either $\mathrm{S}-\mathrm{O}$ or $\mathrm{U}-\mathrm{O}$ travel behavior) is considered to be robust if the index $\mathcal{I}^{\gamma}$ is low. This means that the relative total cost does not change much and, hence, the transportation network may be viewed as being more robust than if the relative total cost is small. 


\section{Remark}

It is worth noting the relationship between the price of anarchy (cf. Roughgarden, 2005, and the references therein), which is denoted by $\rho$, and is defined as:

$$
\rho=\frac{T C_{U-O}}{T C_{S-O}}
$$

and the ratio of the two proposed robustness indices. Observe that $\rho$ captures the relationship between total costs across distinct behavioral principles whereas the indices (14a) and (14b) are focused on the degradation of network performance within U-O or S-O behavior. Nevertheless, we have the following relationship between the ratio of the two indices and the price of anarchy:

$$
\frac{I_{S-O}^{\gamma}}{I_{U-O}^{\gamma}}=\frac{\left[T C_{S-O}^{\gamma}-T C_{S-O}\right]}{\left[T C_{U-O}^{\gamma}-T C_{U-O}\right]} \times \rho
$$

The term preceding the price of anarchy in (16) may be less than 1 , greater than 1 , or equal to 1 , depending upon the network and data. 


\section{Theoretical Results}

In this Section, we derive some theoretical results. In particular, we first prove that for certain networks of special structure, and with links characterized by user link cost functions of BPR form, and with identical free flow travel terms, the relative total cost index is identical under the $\mathrm{U}-\mathrm{O}$ and the $\mathrm{S}-\mathrm{O}$ flow patterns. We then show that for the same network topologies and with user link cost functions also of BPR form, but linear, that the relative total cost index under the U-O flow pattern can be obtained via an explicit formula. In addition, we derive an upper bound for the relative total cost index under the U-O flow pattern. Finally, we derive an upper bound for the relative total cost index for transportation network robustness, under the S-O flow pattern, for any transportation network in the case of user link cost functions of BPR form.

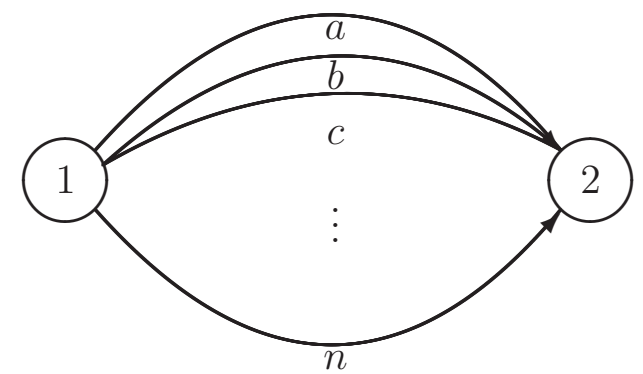

Figure 1: Networks for Theorems 1 and 2

\section{Theorem 1}

Consider a network consisting of two nodes 1 and 2 as in Fig. 1, which are connected by $n$ parallel links. If the free-flow term, $t_{a}^{0}, \forall a \in L$, is the same for all links $a \in L$ in the BPR link cost function (cf. (6)), the S-O flow pattern coincides with the U-O flow pattern and, therefore, $\mathcal{I}_{U-O}=\mathcal{I}_{S-O}$.

Proof: Since the free-flow term $t_{a}^{0}, \forall a \in L$, is the same for all the links, let us denote it as $t^{0}$. First, we show that any U-O flow pattern is also a S-O flow pattern. The reverse can be established in a similar manner. We distinguish between two cases.

\section{Case 1: not all links are used}

If not all links are used then we know that there is at least one link with zero flow.

Select any two links $a, b \in L$ such that $f_{a}^{*}>0$ and $f_{b}^{*}=0$ under the U-O condition. We must have that $c_{a}\left(f_{a}^{*}\right)=\lambda_{w} \leq c_{b}\left(f_{b}^{*}\right)$, where $\lambda_{w}$ is the equilibrium travel disutility for the 
O/D pair $w=(1,2)($ cf. (9)). Hence, we have the following relationships:

$$
\begin{gathered}
t_{a}^{0}\left[1+\left(\frac{f_{a}^{*}}{u_{a}}\right)^{\beta}\right] \leq t_{b}^{0}\left[1+\left(\frac{f_{b}^{*}}{u_{b}}\right)^{\beta}\right] \Longleftrightarrow t^{0}\left[1+\left(\frac{f_{a}^{*}}{u_{a}}\right)^{\beta}\right] \leq t^{0}\left[1+\left(\frac{f_{b}^{*}}{u_{b}}\right)^{\beta}\right] \Longleftrightarrow\left(\frac{f_{a}^{*}}{u_{a}}\right)^{\beta} \leq\left(\frac{f_{b}^{*}}{u_{b}}\right)^{\beta} \\
\Longleftrightarrow t^{0}+t^{0}(\beta+1)\left(\frac{f_{a}^{*}}{u_{a}}\right)^{\beta} \leq t^{0}+t^{0}(\beta+1)\left(\frac{f_{b}^{*}}{u_{b}}\right)^{\beta} \Longleftrightarrow t_{a}^{0}+t_{a}^{0}(\beta+1)\left(\frac{f_{a}^{*}}{u_{a}}\right)^{\beta} \leq t_{b}^{0}+t_{b}^{0}(\beta+1)\left(\frac{f_{b}^{*}}{u_{b}}\right)^{\beta}
\end{gathered}
$$

where the last inequality is exactly the S-O condition (cf. (12)). Therefore, $f_{a}^{*}$ and $f_{b}^{*}$ are also the S-O link flow patterns. Since links $a$ and $b$ were chosen arbitrarily, we can conclude that a U-O flow pattern is also a S-O flow pattern.

\section{Case 2: all links are used}

If all the links are used, under the U-O condition, we must have that

$$
\begin{gathered}
\lambda_{w}=t_{a}^{0}\left[1+\left(\frac{f_{a}^{*}}{u_{a}}\right)^{\beta}\right]=t_{b}^{0}\left[1+\left(\frac{f_{b}^{*}}{u_{b}}\right)^{\beta}\right]=\cdots=t_{n}^{0}\left[1+\left(\frac{f_{n}^{*}}{u_{n}}\right)^{\beta}\right] \\
\Longleftrightarrow \lambda_{w}=t^{0}\left[1+\left(\frac{f_{a}^{*}}{u_{a}}\right)^{\beta}\right]=t^{0}\left[1+\left(\frac{f_{b}^{*}}{u_{b}}\right)^{\beta}\right]=\cdots=t^{0}\left[1+\left(\frac{f_{n}^{*}}{u_{n}}\right)^{\beta}\right] \\
\Longleftrightarrow\left(\frac{f_{a}^{*}}{u_{a}}\right)^{\beta}=\left(\frac{f_{b}^{*}}{u_{b}}\right)^{\beta}=\cdots=\left(\frac{f_{n}^{*}}{u_{n}}\right)^{\beta} \\
\Longleftrightarrow t^{0}+t^{0}(\beta+1)\left(\frac{f_{a}^{*}}{u_{a}}\right)^{\beta}=t^{0}+t^{0}(\beta+1)\left(\frac{f_{b}^{*}}{u_{b}}\right)^{\beta}=\cdots=t^{0}+t^{0}(\beta+1)\left(\frac{f_{n}^{*}}{u_{n}}\right)^{\beta} \\
\Longleftrightarrow t_{a}^{0}+t_{a}^{0}(\beta+1)\left(\frac{f_{a}^{*}}{u_{a}}\right)^{\beta}=t_{b}^{0}+t_{b}^{0}(\beta+1)\left(\frac{f_{b}^{*}}{u_{b}}\right)^{\beta}=\cdots=t_{n}^{0}+t_{n}^{0}(\beta+1)\left(\frac{f_{n}^{*}}{u_{n}}\right)^{\beta}
\end{gathered}
$$

where the last line of (18) shows that $f_{a}^{*}, f_{b}^{*}, \cdots, f_{n}^{*}$ are also S-O link flows (cf. (12)).

By combining the results of Case 1 and Case 2 and from the definitions of $T C_{U-O}, T C_{U-O}^{\gamma}$, $T C_{S-O}$, and $T C_{S-O}^{\gamma}$, we then have that

$$
T C_{U-O}=T C_{S-O} \text { and } T C_{U-O}^{\gamma}=T C_{S-O}^{\gamma}
$$

From the definitions of $\mathcal{I}_{U-O}^{\gamma}(\mathrm{cf} .(14 \mathrm{a}))$ and $\mathcal{I}_{S-O}^{\gamma}(\mathrm{cf} .(14 \mathrm{~b}))$, we conclude that $\mathcal{I}_{U-O}^{\gamma}=$ $\mathcal{I}_{S-O}^{\gamma}$, which completes the proof.

\section{Theorem 2}

Consider a network consisting of two nodes 1 and 2 as in Fig. 1, which are connected by $n$ parallel links. Assume that the associated BPR link cost functions (cf. (6)) have $\beta=1$. Furthermore, assume that there are positive flows on all the links at both the original and 
the partially degraded capacity levels given, respectively, by $u$ and $\gamma u$. Then the relative total cost index under the $U$-O flow pattern is given by the explicit formula:

$$
\mathcal{I}_{U-O}^{\gamma}=\left(\frac{\gamma U+k d_{w}}{\gamma U+k \gamma d_{w}}-1\right) \times 100 \%,
$$

where $d_{w}$ is the given demand for $O / D$ pair $w=(1,2)$ and $U \equiv u_{a}+u_{b}+\cdots+u_{n}$. Moreover, the network robustness $\mathcal{I}_{U-O}^{\gamma}$ is bounded from above by $\frac{1-\gamma}{\gamma} \times 100 \%$.

Proof: Clearly, since there is only a single O/D pair, we have that:

$$
\frac{T C_{U-O}^{\gamma}}{T C_{U-O}}=\frac{d_{w} \times \lambda_{w}^{\gamma}}{d_{w} \times \lambda_{w}}=\frac{\lambda_{w}^{\gamma}}{\lambda_{w}}
$$

where $\lambda_{w}^{\gamma}$ denotes the incurred equilibrium travel disutility for travelers between O/D pair $w$ under the capacity retention ratio $\gamma$.

Due to the special structure of the network as well as the assumption that there are positive flows on all the links before and after the capacity reduction, by referring to the transportation network equilibrium conditions (9), we can write $\lambda_{w}$ and $\lambda_{w}^{\gamma}$ explicitly as follows:

$$
\lambda_{w}=t_{a}^{0}\left(1+k \frac{f_{a}^{*}}{u_{a}}\right)=t_{b}^{0}\left(1+k \frac{f_{b}^{*}}{u_{b}}\right)=\ldots=t_{n}^{0}\left(1+k \frac{f_{n}^{*}}{u_{n}}\right),
$$

where $f_{a}^{*}, f_{b}^{*} \ldots f_{n}^{*}$ are the equilibrium link flows under the link capacities: $u_{a}, u_{b}, \ldots, u_{n}$, respectively, and

$$
\lambda_{w}^{\gamma}=t_{a}^{0}\left(1+k \frac{f_{a}^{* *}}{\gamma u_{a}}\right)=t_{b}^{0}\left(1+k \frac{f_{b}^{* *}}{\gamma u_{b}}\right)=\ldots=t_{n}^{0}\left(1+k \frac{f_{n}^{* *}}{\gamma u_{n}}\right),
$$

where $f_{a}^{* *}, f_{b}^{* *}, \ldots, f_{n}^{* *}$ are the equilibrium link flows under the link capacities: $\gamma u_{a}, \gamma u_{b}, \ldots, \gamma u_{n}$, respectively.

Hence, we have that

$$
\begin{gathered}
\frac{T C_{U-O}^{\gamma}}{T C_{U-O}}=\frac{\lambda_{w}^{\gamma}}{\lambda_{w}}=\frac{t_{a}^{0}\left(1+k \frac{f_{a}^{* *}}{\gamma u_{a}}\right)}{t_{a}^{0}\left(1+k \frac{f_{a}^{*}}{u_{a}}\right)} \\
=\frac{t_{b}^{0}\left(1+k \frac{f_{b}^{* *}}{\gamma u_{b}}\right)}{t_{b}^{0}\left(1+k \frac{f_{b}^{*}}{u_{b}}\right)}=\cdots=\frac{t_{n}^{0}\left(1+k \frac{f_{n}^{* *}}{\gamma u_{n}}\right)}{t_{n}^{0}\left(1+k \frac{f_{n}^{*}}{u_{n}}\right)},
\end{gathered}
$$

which yields

$$
\frac{T C_{U-O}^{\gamma}}{T C_{U-O}}=\frac{\left(1+k \frac{f_{a}^{* *}}{\gamma u_{a}}\right)+\left(1+k \frac{f_{b}^{* *}}{\gamma u_{b}}\right)+\cdots+\left(1+k \frac{f_{n}^{* *}}{\gamma u_{n}}\right)}{\left(1+k \frac{f_{a}^{*}}{u_{a}}\right)+\left(1+k \frac{f_{b}^{*}}{u_{b}}\right)+\cdots+\left(1+k \frac{f_{n}^{*}}{u_{n}}\right)}
$$

After some simplification and from the fact that $f_{a}^{*}+f_{b}^{*}+\ldots+f_{n}^{*}=f_{a}^{* *}+f_{b}^{* *}+\ldots+f_{n}^{* *}=d_{w}$, we obtain

$$
\frac{T C_{U-O}^{\gamma}}{T C_{U-O}}=\frac{\gamma U+k d_{w}}{\gamma U+k \gamma d_{w}} .
$$


From the definition of $\mathcal{I}_{U-O}^{\gamma}$, we have that

$$
\mathcal{I}_{U-O}^{\gamma}=\left(\frac{T C_{U-O}^{\gamma}}{T C_{U-O}}-1\right) \times 100 \%=\left(\frac{\gamma U+k d_{w}}{\gamma U+k \gamma d_{w}}-1\right) \times 100 \%,
$$

which is exactly the form of (20).

To show the upper bound of $\mathcal{I}_{U-O}^{\gamma}$, we can rearrange (26b) to get the following form:

$$
\mathcal{I}_{U-O}^{\gamma}=\left[\frac{\gamma+k \frac{d_{w}}{U}}{\gamma\left(1+k \frac{d_{w}}{U}\right)}-1\right] \times 100 \%
$$

Since $\gamma \in(0,1]$, we have the following:

$$
\mathcal{I}_{U-O}^{\gamma} \leq\left[\frac{1+k \frac{d_{w}}{U}}{\gamma\left(1+k \frac{d_{w}}{U}\right)}-1\right] \times 100 \%=\frac{1-\gamma}{\gamma} \times 100 \%,
$$

which completes the proof.

Now we relax the assumptions in Theorem 2 in that:

1. we do not require the network to be fully loaded before and after the capacity reduction;

2. we do not assume that $\beta=1$; and

3. we do not assume that the network has any special structure.

We now derive a general upper bound for $\mathcal{I}_{S-O}^{\gamma}$ in the following theorem under the above relaxed assumptions.

\section{Theorem 3}

The upper bound for $\mathcal{I}_{S-O}^{\gamma}$ for a transportation network with BPR link cost functions is $\frac{1-\gamma^{\beta}}{\gamma^{\beta}} \times 100 \%$.

Proof: From the definition of $T C_{S-O}$, we have that

$$
T C_{S-O}=\sum_{a \in L} t_{a}^{0}\left[1+k\left(\frac{\hat{f}_{a}}{u_{a}}\right)^{\beta}\right] \times \hat{f}_{a}
$$

where $\hat{f}_{a}, \forall a \in L$, is the S-O link flow pattern under the original link capacities $u$.

Since $\gamma \in(0,1]$ and we assume that $t_{a}^{0}>0, \forall a \in L$,

$$
\frac{1}{\gamma^{\beta}} T C_{S-O} \geq \sum_{a \in L} t_{a}^{0}\left[1+k\left(\frac{\hat{f}_{a}}{\gamma u_{a}}\right)^{\beta}\right] \times \hat{f}_{a} .
$$


Furthermore, denote the S-O link flow pattern under the capacity retention ratio $\gamma$ as $\widetilde{f_{a}}, \forall a \in L$. Then, from the definition of $T C_{S-O}^{\gamma}$, we obtain

$$
\sum_{a \in L} t_{a}^{0}\left[1+k\left(\frac{\hat{f}_{a}}{\gamma u_{a}}\right)^{\beta}\right] \times \hat{f}_{a} \geq \sum_{a \in L} t_{a}^{0}\left[1+k\left(\frac{\widetilde{f_{a}}}{\gamma u_{a}}\right)^{\beta}\right] \times \widetilde{f_{a}}=T C_{S-O}^{\gamma} .
$$

By combining (30) and (31), we obtain

$$
\frac{1}{\gamma^{\beta}} T C_{S-O} \geq T C_{S-O}^{\gamma} \Longleftrightarrow \frac{T C_{S-O}^{\gamma}}{T C_{S-O}} \leq \frac{1}{\gamma^{\beta}}
$$

Hence,

$$
\mathcal{I}_{S-O}^{\gamma} \leq \frac{1-\gamma^{\beta}}{\gamma^{\beta}} \times 100 \%
$$

We have, thus, established the Theorem. 


\section{Numerical Examples}

In this Section we present several numerical examples for which the relative total cost index is computed in the case of alternative travel behaviors.

We utilized the projection method (cf. Dafermos, 1980, and Nagurney, 1999) with the embedded Dafermos and Sparrow (1969) equilibration algorithm (see also, e.g., Nagurney, 1984) to compute the U-O and S-O solutions, respectively. Then, based on the computed respective solutions, we determined the network total cost according to (8) and the relative total cost indices under the user-optimizing and system-optimizing flow patterns according to (14a) and (14b). The computational schemes were implemented in MATLAB. In the case of the large-scale Example 3, we also implemented the column generation algorithm (cf. Leventhal, Nemhauser, and Trotter, 1973) to generate paths, as needed.

\section{Example 1}

Consider the following simple transportation network as shown in Figure 2. There are two $\mathrm{O} / \mathrm{D}$ pairs, namely, $w_{1}=(1,3)$ and $w_{2}=(1,4)$. The demands for the two O/D pairs are: $d_{w_{1}}=10$ and $d_{w_{2}}=20$. The paths connecting O/D pair $w_{1}$ are: $p_{1}=(a, b)$ and $p_{2}=d$. The paths connecting $\mathrm{O} / \mathrm{D}$ pair $w_{2}$ are: $p_{3}=(a, c)$ and $p_{4}=e$. The capacities for links $a, b, c, d$, and $e$ are: $100,50,60,10$, and 20, respectively. Let $t_{0}$ and $k$ (cf. (6)) be identical for all the links and equal to 10 and 1, respectively. The BPR link cost functions for the links in Fig. 2 are, hence, given by:

$$
\begin{gathered}
c_{a}\left(f_{a}\right)=10\left[1+\left(\frac{f_{a}}{100}\right)^{\beta}\right], \quad c_{b}\left(f_{b}\right)=10\left[1+\left(\frac{f_{b}}{50}\right)^{\beta}\right], \\
c_{c}\left(f_{c}\right)=10\left[1+\left(\frac{f_{c}}{60}\right)^{\beta}\right], \quad c_{d}\left(f_{d}\right)=10\left[1+\left(\frac{f_{d}}{10}\right)^{\beta}\right], \quad c_{e}\left(f_{e}\right)=10\left[1+\left(\frac{f_{e}}{20}\right)^{\beta}\right] .
\end{gathered}
$$

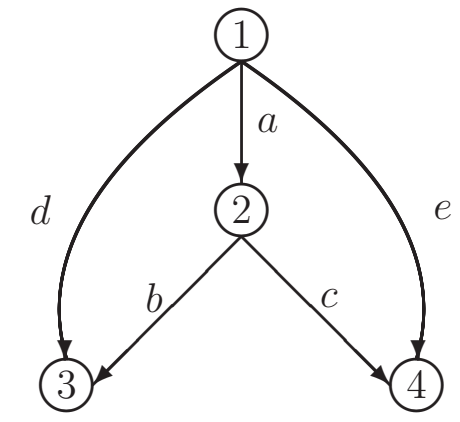

Figure 2: Example 1 Network

Fig. 3 presents the relative total cost index for Example 1 under the U-O flow pattern for $\beta$ values equal to 1, 2, 3, and 4, respectively. Similarly, Fig. 4 presents the index for Example 1 under the $\mathrm{S}-\mathrm{O}$ flow pattern for $\beta$ values equal to $1,2,3$, and 4 , respectively. 


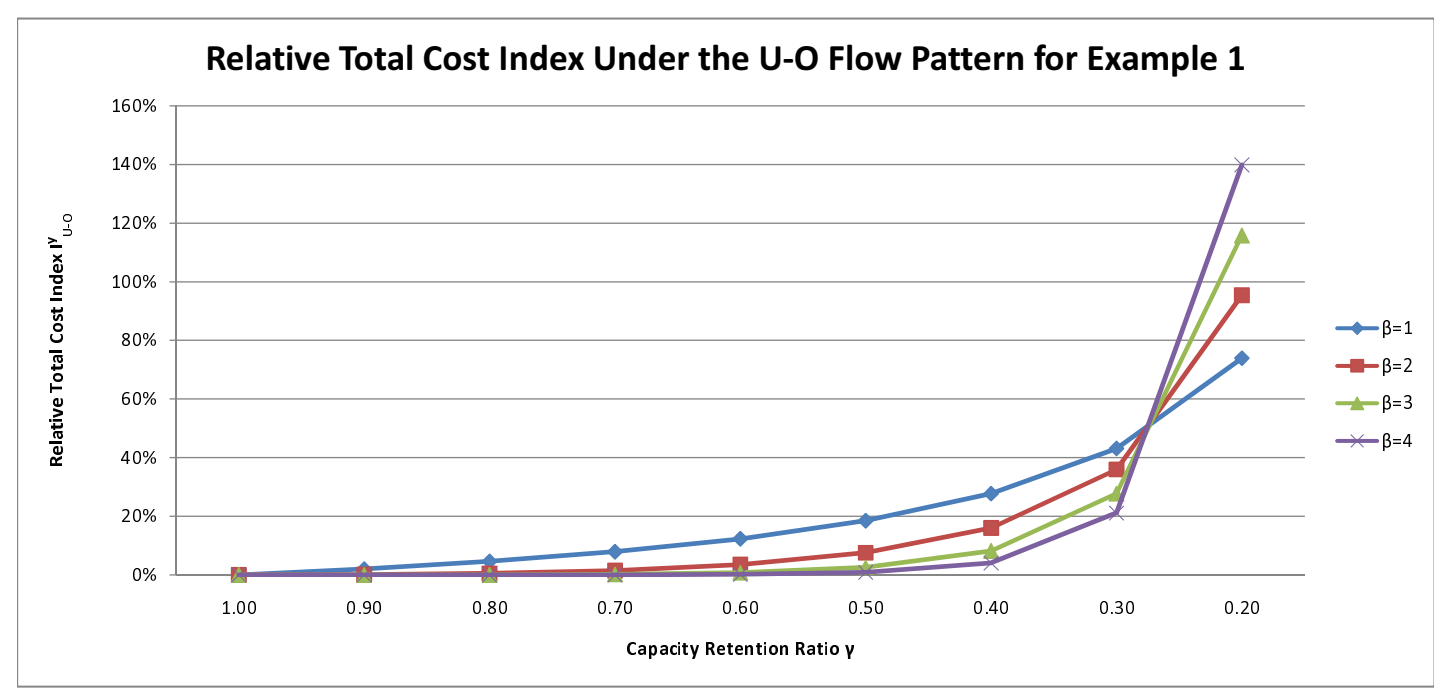

Figure 3: Relative Total Cost Index Under the U-O Flow Pattern for Example 1

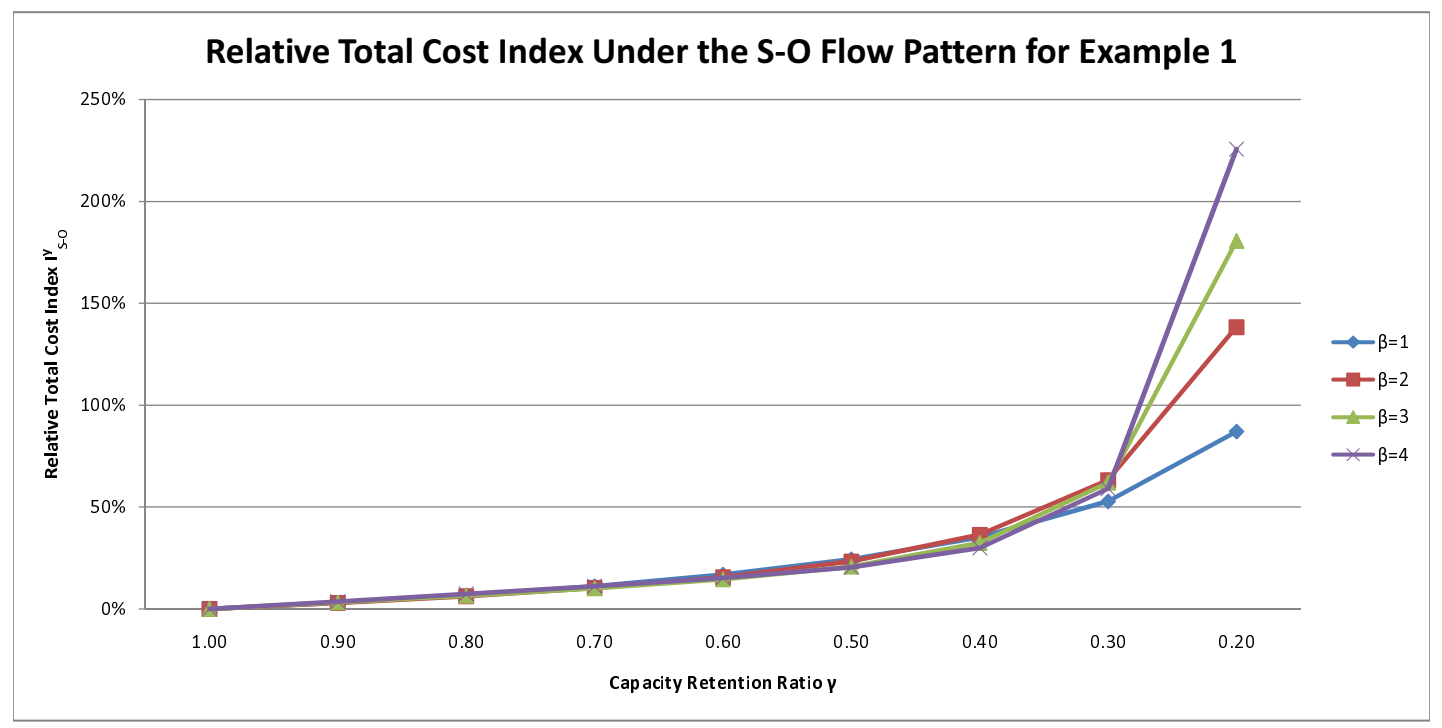

Figure 4: Relative Total Cost Index Under the S-O Flow Pattern for Example 1 
Interestingly, we can see from Figs. 3 and 4 that, although under the S-O flow pattern, the total cost of the entire transportation network is minimized, the relative total cost index under the $\mathrm{U}-\mathrm{O}$ flow pattern is lower given the same capacity retention ratio $\gamma$. Therefore, this transportation network under the U-O solution concept is more robust than under the S-O solution concept.

\section{Example 2: The Braess Network}

We now consider the Braess Paradox network after the addition of a new link $e$ and as depicted in Fig. 5 (see also Braess, 1968, and Braess, Nagurney, and Wakolbinger, 2005). There are four nodes: $1,2,3$, 4; five links: $a, b, c, d, e$; and a single O/D pair $w_{1}=(1,4)$. There are, hence, three paths connecting the single O/D pair, which are denoted, respectively, by: $p_{1}=(a, c), p_{2}=(b, d)$, and $p_{3}=(a, e, d)$.

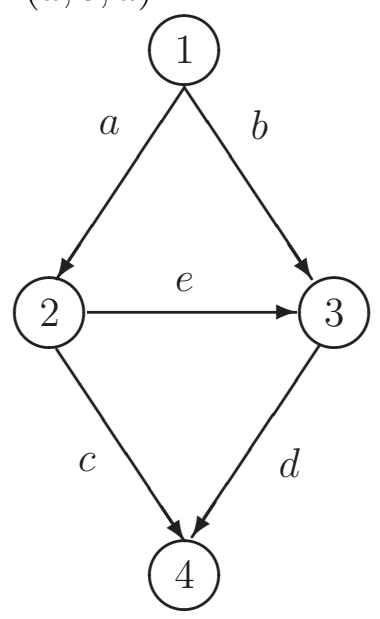

Figure 5: The Braess Network Topology

Instead of using the original Braess (1968) link cost functions, since they are not of BPR form, we construct a set of BPR functions under which the Braess Paradox still occurs (without any capacity reduction). We assume that $k=1$. Let $t_{a}^{0}=t_{d}^{0}=1 ; t_{b}^{0}=t_{c}^{0}=50$, and $t_{e}^{0}=10$. Furthermore, let $u_{a}=u_{d}=20 ; u_{b}=u_{c}=50$, and $u_{e}=100$. The user link cost functions are, thus, given by:

$$
\begin{gathered}
c_{a}\left(f_{a}\right)=1+\left(\frac{f_{a}}{20}\right)^{\beta}, \quad c_{b}\left(f_{b}\right)=50\left[1+\left(\frac{f_{b}}{50}\right)^{\beta}\right], \\
c_{c}\left(f_{c}\right)=50\left[1+\left(\frac{f_{b}}{50}\right)^{\beta}\right], \quad c_{d}\left(f_{d}\right)=1+\left(\frac{f_{d}}{20}\right)^{\beta}, \\
c_{e}\left(f_{e}\right)=10\left[1+\left(\frac{f_{e}}{100}\right)^{\beta}\right] .
\end{gathered}
$$

The demand is given by $d_{w_{1}}=110$. Fig. 6 presents the relative total cost index for the Braess network under the U-O flow pattern for $\beta$ values equal to $1,2,3$, and 4 , respectively. 
Similarly, Fig. 7 presents the index for the Braess network under the S-O flow pattern for $\beta$ values equal to $1,2,3$, and 4 , respectively.

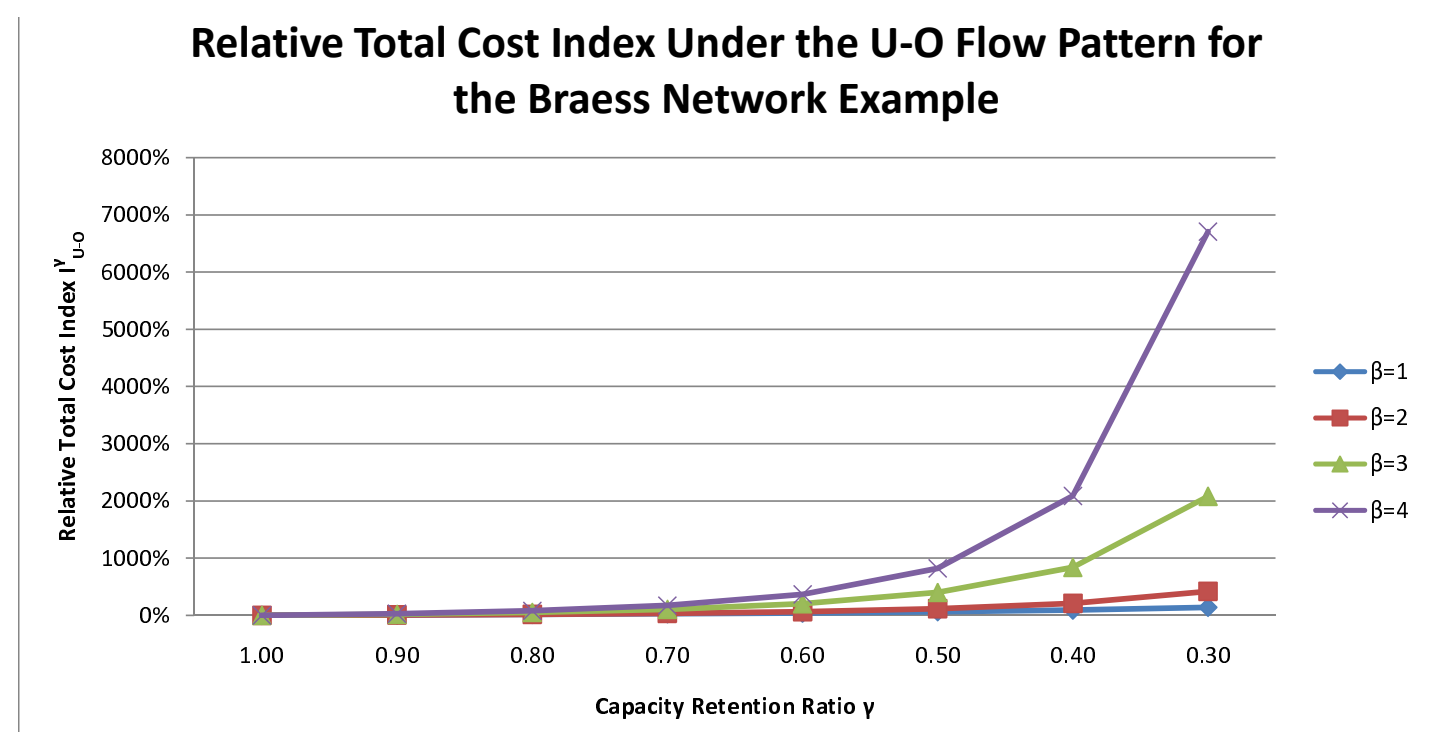

Figure 6: Relative Total Cost Index Under the U-O Flow Pattern for the Braess Network Example

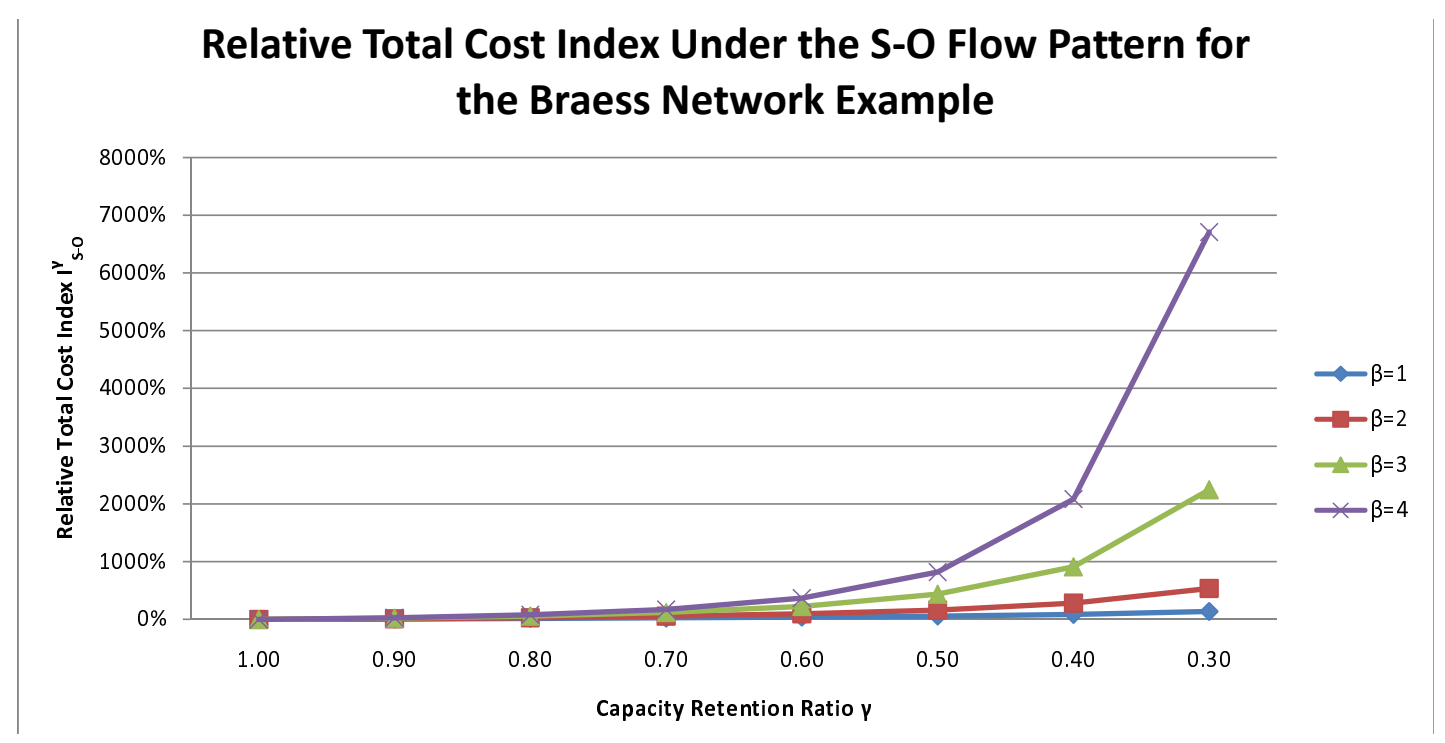

Figure 7: Relative Total Cost Index Under the S-O Flow Pattern for the Braess Network Example

In the above example, when $\beta=2$ or 3 , the relative total cost index under the U-O flow pattern is lower than that under the S-O flow pattern, which means that the network with user behavior associated with the $\mathrm{U}-\mathrm{O}$ condition is more robust when $\beta=2$ or 3 . 


\section{Example 3: The Sioux-Falls Network}

We now consider the Sioux-Falls network (cf. LeBlanc, Morlok, and Pierskalla, 1975).

The network topology is shown in Figure 8.

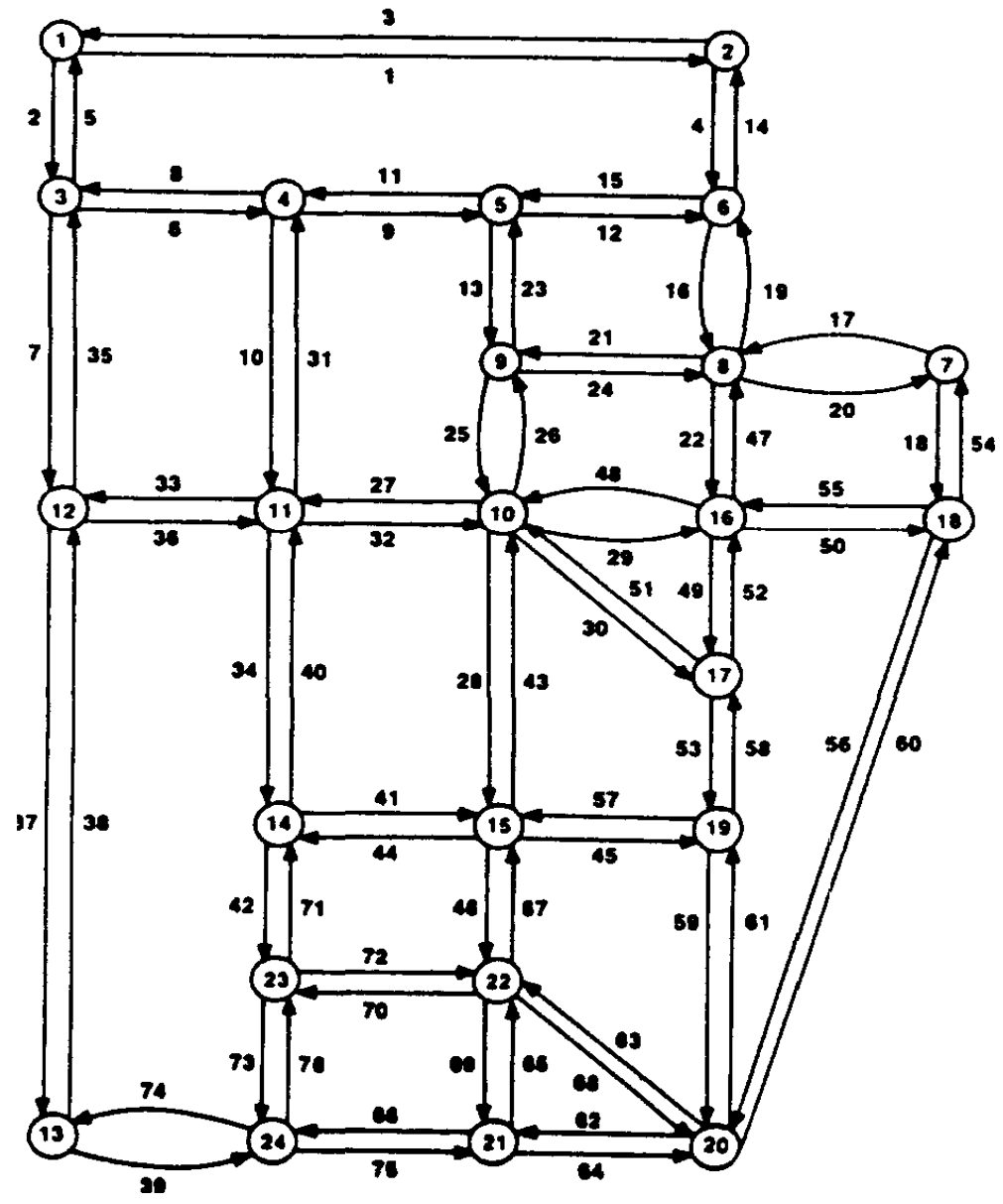

Figure 8: The Sioux-Falls Network (Friesz et al., 1994)

There are 528 O/D pairs, 24 nodes, and 76 links in the Sioux-Falls network. For the relevant data see LeBlanc, Morlok, and Pierskalla (1975) and the transportation network datasets maintained by Bar-Gera: http://www.bgu.ac.il/ bargera/tntp/.

Fig. 9 presents the relative total cost index for the Sioux-Falls network under the U-O flow pattern for $\beta$ values equal to 1, 2, 3, and 4, respectively. Similarly, Fig. 10 presents the index for the Sioux-Falls network under the S-O flow pattern for $\beta$ values equal to $1,2,3$, and 4 , respectively. 


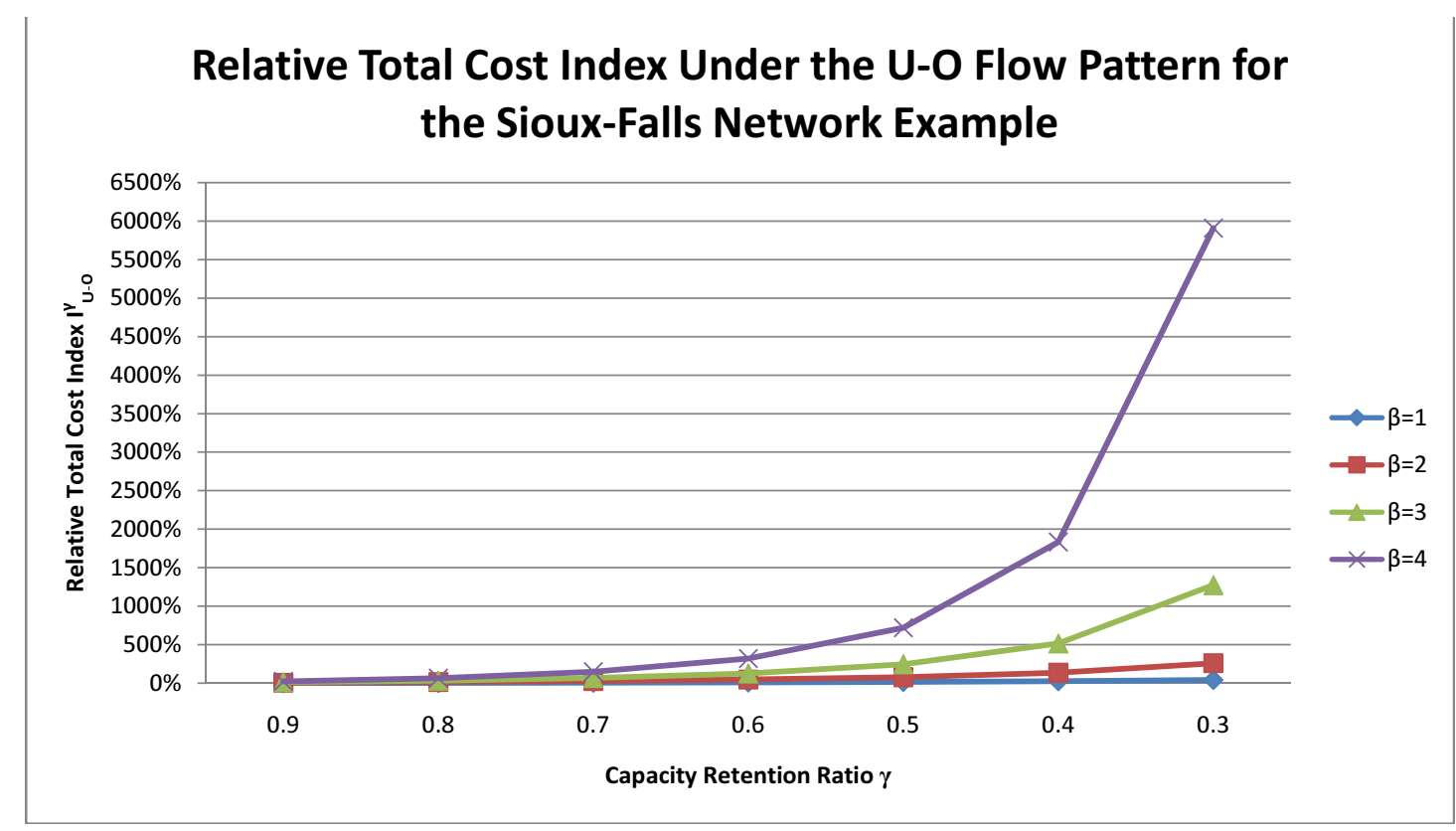

Figure 9: Relative Total Cost Index Under the U-O Flow Pattern for the Sioux-Falls Network Example

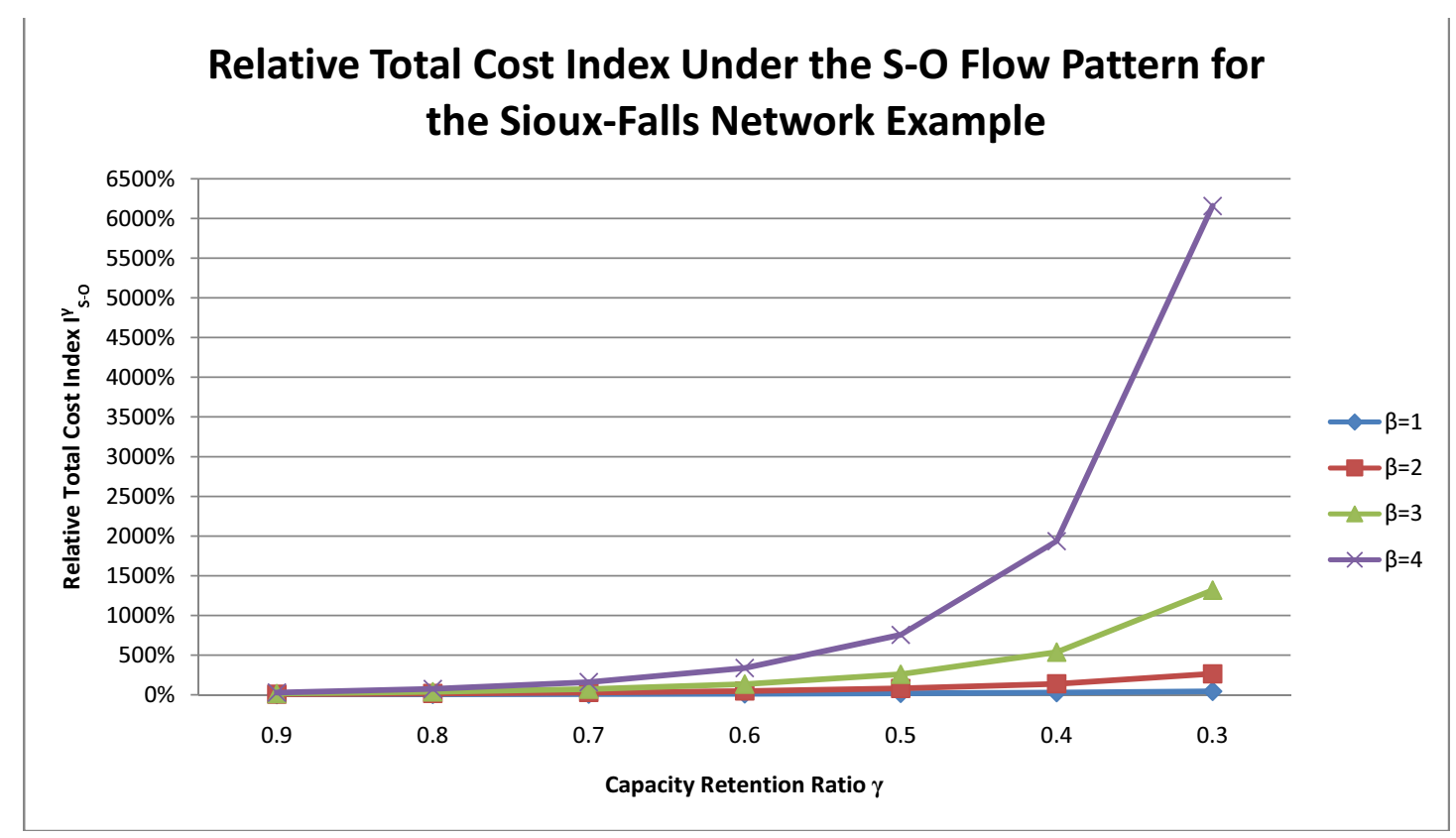

Figure 10: Relative Total Cost Index Under the S-O Flow Pattern for the Sioux-Falls Network Example

To compare the two indices under different $\beta$ values and capacity retention ratios, we exhibit the ratio of $I_{S-O}^{\gamma}$ to $I_{U-O}^{\gamma}$ in Fig. 11 below. 


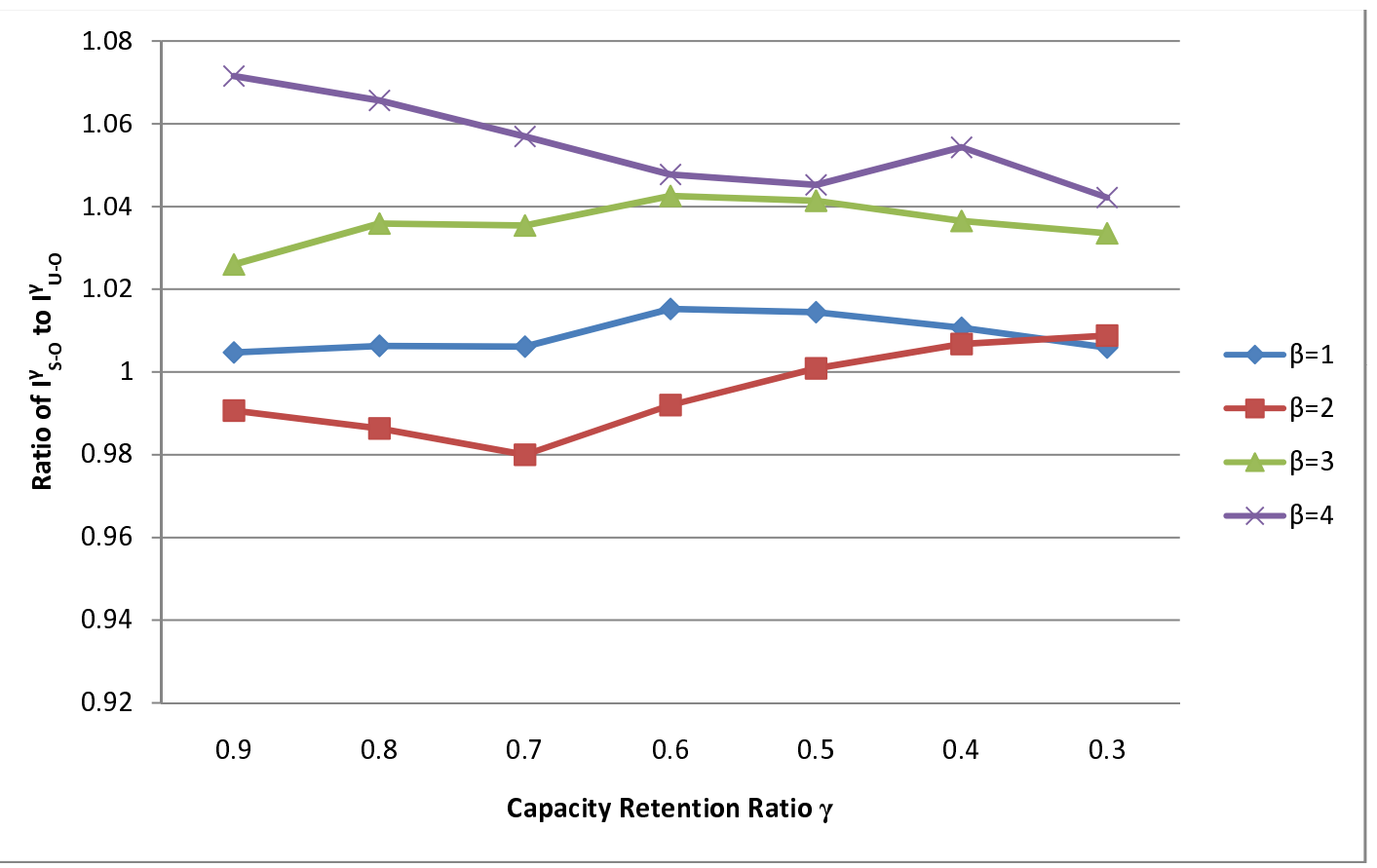

Figure 11: Ratio of $I_{S-O}^{\gamma}$ to $I_{U-O}^{\gamma}$ for the Sioux-Falls Network Example

From Fig. 11, we can see that the Sioux-Falls network is always more robust under U-O behavior except when $\beta$ is equal to 2 and the capacity retention ratio is between 0.5 and 0.9 .

One can also see that Theorem 3 applies to both Examples 1, 2 and 3 in that it provides an upper bound on $\mathcal{I}_{S-O}^{\gamma}$ as reflected by the results in Figures 4, 7 and 10.

\section{Summary and Conclusions}

In this paper, we presented two relative total cost indices to analyze transportation network robustness associated with either system-optimal or user-optimal user behavior. Assuming that the user link cost functions for the transportation network are of BPR form, we examined the impacts of the degradation of the links in terms of capacity reductions on the relative total cost in the network at the original capacities and at the degraded capacities. We established new theoretical results and also provided numerical examples.

For future research, we plan on assessing the robustness of transportation networks with asymmetric BPR-type link cost functions (see http://www.bgu.ac.il/ bargera/tntp/ for examples of such link cost functions) and with multiple modes of transportation. Furthermore, we would also like to study the robustness of transportation networks in the case of stochastic costs (and demands). Of course, the derivation of additional theoretical results would 
also be valuable.

\section{Acknowledgments}

This research was supported by the John F. Smith Memorial Fund at the University of Massachusetts at Amherst. This support is gratefully appreciated. The authors are grateful to the two anonymous reviewers and the Associate Editor for helpful comments and suggestions on the earlier version of this paper.

\section{References}

Albert, R., Jeong, H., Barabási, A. L., 2000. Error and attack tolerance of complex networks. Nature 406, 378-382.

American Society of Civil Engineers, 2005. Report card for America's infrastructure.

Bazaraa, M. S, Sherali, H. D, Shetty, C. M., 1993. Nonlinear programming: theory and algorithms. John Wiley \& Sons, New York.

Beckmann, M. J., McGuire, C. B., Winsten, C. B., 1956. Studies in the economics of transportation. Yale University Press, New Haven, Connecticut.

Boyce, D. E., Mahmassani, H. S., Nagurney, A., 2005. A retrospective on Beckmann, McGuire and Winsten's Studies in the economics of transportation. Papers in Regional Science, 84, 85-103.

Braess, D., 1968. Uber ein paradoxon aus der verkehrsplanung. Unternehmenforschung 12, 258-268.

Braess, D., Nagurney, A., Wakolbinger, T., 2005. On a paradox of traffic planning, translation of the 1968 article by Braess. Transportation Science 39, 446-450.

Bureau of Public Roads, 1964. Traffic assignment manual. U.S. Department of Commerce, Urban Planning Division, Washington, DC.

Dafermos, S., 1980. Traffic equilibrium and variational inequalities. Transportation Science, $14,42-54$.

Dafermos, S. C., Sparrow, F. T., 1969. The traffic assignment problem for a general network. Journal of Research of the National Bureau of Standards 73B, 91-118.

Environment News Service, January 22, 2008. Governors, mayor form coalition to rebuild ailing U.S. infrastructure. http://www.ens-newswire.com/ens/jan2008/2008-01-22-02.asp. 
Friesz, T. L., David, B., Mehta, N. J., Tobin, R. L., Ganjalizadeh, S., 1994. Day-to-day dynamic network disequilibria and idealized traveler information systems. Operations Research $42,1120-1136$.

Federal Highway Administration, 2006. An initial assessment of freight bottlenecks on highways, Washington, DC.

Gribble, S. D., 2001. Robustness in complex systems. Proceedings of the 8th Workshop on Hot Topics in Operating Systems (HotOS-VIII), 21-26.

Holmgren, A. J., 2007. A framework for vulnerability assessment of electric power systems. In: Murray, A., Grubesic, T. (Eds), Reliability and vulnerability in critical infrastructure: a quantitative geographic perspective, Springer, New York, pp. 31-55.

Institute of Electrical and Electronics Engineers 1990. IEEE standard computer dictionary: a compilation of IEEE standard computer glossaries. New York.

Jeanneret, M., 2006. Federal government freight bottleneck report highlights America's growing transportation infrastructure crisis.

http://artba.com/news/press_releases/2006/02-03-06.htm.

LeBlanc, L. J., Morlok, E. K., Pierskalla, W. P., 1975. An efficient approach to solving the road network equilibrium traffic assignment problem. Transportation Research B 9, 309-318.

Leventhal, T., Nemhauser, G., Trotter, L., 1973. A column generation algorithm for optimal traffic assignment. Transportation Science 7, 168-176.

Nagurney, A., 1984. Computational comparisons of algorithms for general asymmetric traffic equilibrium problems with fixed and elastic demands. Transportation Research $B$ 18, 469485.

Nagurney, A., 1999. Network economics: a variational inequality approach (2nd edition). Kluwer Academic Publishers, Dordrecht, The Netherlands.

Nagurney, A., 2000. Sustainable transportation networks. Edward Elgar Publishing, Cheltenham, England.

Nagurney, A., 2006. Supply chain network economics: dynamics of prices, flows, and profits. Edward Elgar Publishing, Cheltenham, England.

National Bridge Inventory Database, 2005. http://nationalbridges.com/.

Nagurney, A., Liu, Z., Cojocaru, M. G., and Daniele, P., 2007. Static and dynamic trans- 
portation network equilibrium reformulations of electric power supply chain networks with known demands. Transportation Research E 43, 624-646.

Nagurney, A., Qiang, Q., 2007a. A network efficiency measure for congested networks. Europhysics Letters 79, 38005, 1-5.

Nagurney, A., Qiang, Q., 2007b. Robustness of transportation networks subject to degradable links. Europhysics Letters 80, 68001, 1-6.

Perakis, G., 2007. The "price of anarchy" under nonlinear and asymmetric costs. Mathematics of Operations Research 23, 614-628.

Roughgarden, T., 2005. Selfish routing and the price of anarchy. MIT Press, Cambridge, Massachusetts.

Sakakibara, H., Kajitani, Y., Okada, N., 2004. Road network robustness for avoiding functional isolation in disasters. Journal of Transportation Engineering 130, 560-567.

Scott, D. M., Novak, D., Aultman-Hall, L., Guo, F., 2006. Network robustness index: a new method for identifying critical links and evaluating the performance of transportation networks. Journal of Transport Geography 14, 215-227.

Schillo, M., Bürchert, H., Fischer, K., Klusch, M., 2001. Towards a definition of robustness for market-style open multi-agent systems. Proceedings of 5th International Conference on Autonomous Agents, 75-76.

Sheffi, Y., 1985. Urban transportation networks - equilibrium analysis with mathematical programming methods. Prentice-Hall, Englewood Cliffs, New Jersey.

Smith, M., 1979. Existence, uniqueness, and stability of traffic equilibria. Transportation Research B 13, 259-304.

Wald, M. L., Chang, K., August 3, 2007. Minneapolis bridge had passed inspections. New York Times. http://www.nytimes.com/2007/08/03/us/03safety.html.

Wardrop, J. G., 1952. Some theoretical aspects of road traffic research. Proceedings of the Institution of Civil Engineers, Part II 1, 325-378. 